\title{
Pandemic Pivots in Teacher Education: Creating and Sustaining the New Normal
}

\author{
Christopher G. Clinton, ${ }^{1}$ and Maureen P. Hall ${ }^{2}$ \\ 1,2Department of STEM Education and Teacher Development, University of Massachusetts Dartmouth
}

Cite as: Clinton, C.G. \& Hall, M.P. (2022). Pandemic Pivots in Teacher Education: Creating and Sustaining the New Normal. Metropolitan Universities, 33(1), 3-12. DOI: 10.18060/25572.

This is an open access article distributed under the terms of the Creative Commons Attribution License.

Editor: Valerie L. Holton, Ph.D.

\begin{abstract}
This article documents how one Teacher Preparation Program (TPP) at a medium-sized public university in the northeast responded to the needs of their urban partners during the global COVID-19 pandemic. The adaptation of this school-university partnership involved improving relationships where the TPP is more responsive to the needs of this urban school district. This article showcases how two colleagues created pandemic pivots in their graduate courses for inservice teachers. These pedagogical examples, or pandemic pivots, knit together technology and Social Emotional Learning (SEL) and create a more inclusive and more equitable new normal. Moreover, these pandemic pivots leverage programmatic mechanisms to sustain this new normal. The sustainability and success of this partnership makes use of stakeholder feedback loops which are both programmatic and interpersonal. Through institutionalizing these feedback loops, we create programmatic sustainability with the ability to dynamically meet the needs of our partners. The integration of SEL and technology pedagogical practices illustrated here are the result of programmatic structural changes driven by the voice of our partners, showcasing the design of our TPP.
\end{abstract}

Keywords: pandemic pivots, social emotional learning, SCARF model, lectio divina, sustainability 


\section{Introduction}

As a public research university, the mission of University of Massachusetts Dartmouth (UMassD) is to provide high impact learning experiences and engage in innovative research resulting in personal and lifelong student success. Our Teacher Preparation Programs (TPP) are committed to the preparation of urban educators who have a sensitivity toward multicultural issues, an awareness of the needs of schools, and knowledge of the unique needs and styles of individual learners. The TPP at the UMassD recently shifted its master's in teaching (MAT) programming to focus strategically on developing urban educators. Our primary partner school districts, Fall River Public Schools and New Bedford Public Schools, are the significant stakeholders, and both districts report issues of high teacher turnover rates and student underperformance. The intentional design of the new TPP is a responsive model, one designed to meet our urban partners' needs and focused on long-term teacher retention and improved student success. Almeida and Hall (2021a) write about this partnership showing that "the logistical aspects of this innovation school-university partnership and program are responsive to, rather than in competition with, the realities and professional obligations of in-service teachers" ( $p$. 371). Moreover, our TPP requires thoughtful and skilled professionals to monitor, analyze, and modify their practices in an ongoing process of reflection and action effectively integrating both theory and practice, being responsive to our school partners' needs. This model underlines their commitment to reflective practice and developing culturally aware, effective, urban teacher candidates in their content and practice.

Our initiatives include the Teacher Education Advisory Board (TEAC) at the programmatic level, allowing for continuous two-way communication between our partners and ourselves and the development of instructional consultants positions at the course level. Instructional consultants (IC) are a cornerstone to strengthening and growing the partnership between UMassD and K-12 partners. District leadership are tasked with identifying qualified district personnel to serve as paid ICs collaborate with faculty in the delivery of on-site coursework. ICs work alongside university faculty to co-construct course objectives in alignment with the partner district goals. ICs also collaborate with university faculty in the delivery of MAT courses to ensure course objectives target specific content and pedagogical strategies identified as high impact in the urban classroom. Both the TEAC and the IC roles serve to improve feedback to UMassD's TPP in the development and deployment of the course and program. The TEAC also act effectively as gatekeepers into the profession by selecting candidates for admission and providing input on standards for program completion (Clinton, 2019). The stakeholder composition of the TEAC is critical its sustainability as a feedback mechanism for the partnership. Clinton, (2019) found the greater participation of school-based leadership (teacher leaders) increased the sustainability of the TEAC when compared to other advisory committees whose composition retained a greater number of central office personnel (superintendents). As a result, at UMassD the TEAC is made up of seven out of 11 members are school-based leaders, one is a central office leader, and three are university faculty. The elevation of agency of school- 
based leadership on the TEAC allows for opportunities to initiate and sustain long-term changes and provide a feedback mechanism evaluating changes in the TPP. As district leadership and central office personnel often change, at rates equivalent to $20 \%$ per year in the surrounding partnership districts (Clinton, 2019), having an TEAC who will guide and then benefit from changes in the TPP reinforces a new normal. In this new normal where the traditional power dynamic of the University providing guidance to the school partner, (Clinton, 2019) is reversed and the school-based leadership provide goal setting for the faculty of the TPP. Finally, both the TEAC and ICs operate as professional networks influencing whether teacher candidates obtain positions in the partner schools. This has a positive impact on retention rates (Goldhaber \& Cowan, 2014; Krieg et al., 2016). By leveraging the input from these roles, it provides a feedback loop motivating UMassD on maintaining our school partners' new normal.

Early in the COVID-19 pandemic, a TEAC meeting was convened to address what changes the TPP should be made to better support urban K-12 partners and address their identified needs.

The conversation was structured with a focus on short-term and long-term needs. The short-term needs focused significantly on pedagogical training for both in-service and preservice candidates to specifically meet the diverse needs of students in the new online environment. In addition, the TEAC discussed long-term issues of disruption between school culture and broader community. Students in online environments were feeling disconnected from their learning communities further impeding their academic development. Conversations quickly turned to how the TPP could pivot modifying their own curriculum to include pedagogical strategies in the new online learning landscape while also meeting the identified need SEL and maintaining community and cultural relevancy. It was these targeted conversations around the district identified goals both long and short-term which drove the development of these strategies and their implementation in the TPP.

During the COVID-19 pandemic, both the TEAC and IC roles played essential elements in identifying the changes necessary for the preservice and in-service teachers to be successful in the pandemic classroom. The new normal in this paper represents an ideology of never letting a crisis never letting a crisis pass without consideration of challenges and solutions. The pandemic created new inequities but also significantly underscored pre-existing conditions which had long gone unaddressed by stakeholders. Sustainable initiatives such as TEAC, the ICs, and a more robust integration of social emotional learning with technology served to strengthen the university and urban K-12 relationships' during the pandemic and developed a more supportive and responsive learning community. 


\section{Pandemic Pivots: Movements for Fostering Structural Change}

Although there were many downsides to the pandemic, there are also new insights which emerged. Ailwood and Lee's (2020) insights highlighted that "care is essential to the sustenance of all our lives." They point out the varied responses to COVID-19 lockdowns created significant "educational equity issues" (p. 309). White and McCallum (2021) explored the need for resilience in teaching and learning. During the pandemic, teachers were forced to find new ways to integrate technology, engage their students, continue to develop healthy relationships with their students, and expected to make strides in content learning. The pandemic pivot examples illustrated in this article demonstrate how teacher educators found dynamic ways to inextricably link Social Emotional Learning (SEL) with technology. New learning models, integrating SEL with technology were needed, and these models also must address equity issues raised during the pandemic.

Historically, SEL and technology have been disconnected. SEL, seen as essential to success in teaching and learning, includes self-care, emotion management, and healthy relationship building (Jennings, 2015). Likewise, technology practices, including presentation, gamification, and exploration were centric to success in the online delivery of content. Prior to the pandemic, technology was emerging in importance, but not viewed as critical to instruction. During this pandemic pivot, technology offered the only means to access content and became critical to delivery. SEL skills, when integrated with technology delivery in the classroom, became instrumental to success during the pandemic.

\section{Pandemic Pivot Examples}

The pandemic pivots outlined below were designed to help in-service teachers find success in our graduate courses and demonstrate their learning during the pandemic. We modeled teaching approaches as templates that our teachers could try out and replicate in their own K-12 classrooms. Though we as teacher educators have different content backgrounds; Chris has a doctorate in STEM Education and taught science in K-12, and Maureen has her doctorate in English education and taught English in K-12. We both teach pedagogical courses for our cohorts of urban teachers. What follows are examples of pandemic pivots that each of us innovated over the pandemic in response to the needs of our school partner districts.

\section{Pandemic Pivot 1}

Our school partners tasked our TPP to embed in our coursework more SEL strategies as deployed through technology. What became clear after some discussion with the ICs and TEAC was the need for a framework to allow teacher candidates to be mindful of how different instructional practices impacted students socially and emotionally. The SEL framework employed in this strategy was grounded in David Rock's SCARF model (Rock, 2008). This 
model presents five domains which can have an impact on a person's perception of their own social emotional status within a situation. The domains are Status, which refers to one's sense of importance relative to others; Certainty, which refers to one's need to make clear and accurate predictions about their environment; Autonomy, or the sense of independence one gets having control over events or impact on the outcome of a situation; Relatedness, defined as having a connection to the material and or others; and Fairness, which is the perception of unbiased exchange. These elements, when incorporated into technology and instruction in the classroom, can alleviate most students' social concerns, allowing them great opportunity for classroom engagement (Rock and Tang, 2009). For my pandemic pivot, I used the SCARF model as my theoretical framework and incorporated the website Classcraft into my instructional design for the MAT coursework in response to the urban teachers' needs.

Classcraft is a gamification of a traditional Google Classroom site. Gamification is a multidisciplinary tool which tries to create a motivating, engaging and emotionally positive context for learning. The simplistic Google Classroom interface is gamified, embodying elements of the SCARF framework. One reason for gamification is research indicating participating in the game and learning are similar neurological mechanisms (Lee, and Hammer, 2011). The Classcraft overlay allows for a culturally responsive narrative integrated with classroom competencies and multiple opportunities for students to exercise control over their experience. Many facets of the Classcraft experience align with the SCARF and an SEL framework (Elias, 2018). As Membrive and Armie (2020) point out, the Classcraft environment stimulates engagement in activities related to the content of the course. In addition, Rozhenko, Darzhaniya, Bondar, \& Mirzoian, (2021) found, "psychological climate at the lesson has changed enormously: equanimity, constraint disappeared, interest, 'sparkle in the eyes' appeared" (p.463) when Classcraft was integrated as part of the Google Classroom interface. Both papers directly reference an increase in students motivation linked to their multiple opportunities to positively augment their Status as the game progresses. Competencies and classroom assignments scored on game points allow for greater customization and progression also are linked to positive student outcomes. Because the Classcraft overlay integrates with the existing Google Classroom, the students and teachers have a sense of Certainty around assignments and expectations. Student's progress on various Quests within the program allowing for Autonomy and differentiated instruction. The narrative of the Classcraft experience can be modified by the teacher to create Relatedness and integrate cultural competencies (Elias, 2018). Finally, the expectations and point systems are clear and modifiable to be Fair to students who participate. This flexibility and customization of the overlay allows for greater intrinsic motivation of students in the corresponding course materials and fosters increased social engagement over traditional Google Classroom structures (Bonvin \& Sanchez, 2017). By using the elements of Rock's SCARF model, urban educators can predetermine if the technology integration in their course will adequately meet the social emotional needs of their students.

(C) The Author 2022. Published by the Coalition of Urban and Metropolitan Universities. www.cumuonline.org Metropolitan Universities | DOI 10.18060/25572 | February 15, 2022 
The pandemic pivot is the use of the SCARF framework as an evaluative tool for technology integration to better understand how students' social and emotional responses will be engendered with these tools. Using the SCARF model as a social emotional framework allows practitioners predict how students will interact and potentially respond to the technology tools before their implementation in the classroom. Questions practitioners would ask might include, "Does the interaction with this technology potentially increase the status of the student?"; "Is there significant autonomy in this assignment using this technology tool to allow the students to determine how they reach the outcome? "; "Is the content and the integration of technology enhancing the relatedness of the material to the student?"; and "Are the outcomes when using this technology either a stretch and within reach or even just beyond reach of the students and considered by the students to be a fair measure of their effort?" The SCARF model as a framework serves to allow the practitioner the opportunity to think of points where students would traditionally disconnect from the learning, especially when isolated in an online setting. Using the SCARF model minimizes potential negative interactions between the pedagogical strategies employed by the teachers with their students. The new normal will be using the SCARF framework for multiple pedagogical strategies regardless of the technology employed.

\section{Pandemic Pivot 2}

The co-author teaches a course called EDU 525 Critical Literacies, which has four main themes: Critical Literacies, Social Emotional Learning (SEL), Place-Based Learning (PBL), and Global Citizenship. SEL is one of the district goals from our partner school district in Fall River, Massachusetts.

Over the pandemic, lectio divina was used as a pedagogical tool in EDU 525 over Zoom. Lectio divina is part of a contemplative pedagogy with ancient roots. Lectio divina, as a pandemic pivot, became a conduit for in-service teachers to understand both issues of power and equity, along with how they might modify lectio divina for use with their own students. Lectio divina is an ancient tool for understanding texts. With religious roots, it has adapted secular forms for twenty first-century classrooms (Dalton, Hall, \& Hoyser, 2019). By choosing short passages for discussion, lectio divina was utilized as a communal reading process and "vehicle" for transformative dialogue. Moreover, lectio divina models empathic and dialogic teaching (Tan, 2021) and mindful learning through literacy.

As Dorman (2019) points out, "Contemplative practices such as lectio divina implemented in teacher education allow an integrated, embodied approach for learners to unwind the conditioned mental habits of racism, prejudice, white supremacy, colonization, microaggressions, and other forms of oppression that cause harm and suffering" (p. 72). Through the lectio divina process, students learned how to do deep listening and open themselves to new understandings of difference and possibilities for social action.

(C) The Author 2022. Published by the Coalition of Urban and Metropolitan Universities. www.cumuonline.org Metropolitan Universities | DOI 10.18060/25572 | February 15, 2022 
More specifically, in fall 2020 the co-author collaborated with two education colleagues from UMass Lowell and UMass Boston using lectio divina as an anti-racist pedagogy. In preparation for our session (Bifuh-Ambe, Hall, \& Lopes-Mendes, 2020) in CUMU's Learning \& Sharing Virtual Series, she invited Elizabeth Bifuh-Ambe of UMass Lowell and Tyra Lopes of UMass Boston each to join one of her EDU 525 class sessions when they were using lectio divina as an anti-racist pedagogy. In October 2020, Professor Bifuh-Ambe was a guest and Langston Hughes' poem, "A Dream Deferred " was used as the text for this pedagogical intervention with lectio divina. Together with Professor Bifuh-Ambe, they innovated using lectio divina as both an antiracist pedagogy and a critical literacy pedagogy. As part of the lectio divina step of oratio (response), students were assigned to Zoom breakout rooms with a partner. In the breakout rooms, students were instructed to deep listen to their partner's response. Students brainstormed actions they could take to promote anti-racism and help each other to become social justice allies. Each partner would then share the other's response when returning from the breakout rooms. Students wrote reflections about this experience, and one student shared:

In my experience with using lectio divina to analyze "A Dream Deferred," we were encouraged to take our time in reading and hearing a text, letting it wash over us and settle into the cracks of our consciousness. Through this, we heard the words differently than before: the symbolism and imagery became richer, more relevant and meaningful as we mapped it onto our own experiences, or imagined how they made others feel...Lectio divina allowed for multiple means of expression as well, something that is incredibly important in creating a more inclusive classroom: we listened to the text and our partner's reflections, drew how it made us feel, or sometimes created our own poems in response and read them aloud to the class (Serena Campbell, personal narrative, October 25, 2020).

Campbell experienced literacy as a new window into herself and others. Through lectio divina as a conduit, she experienced another way to build inclusivity. Empathy, as part of SEL, is evident. Students can step into another's shoes and understand their perspective. Teaching understanding through literacy improves all human relations.

Leveraging the technology provided by Zoom, the breakout rooms allowed for deep listening between partners and helped to model and promote SEL for participants. The pandemic pivot of instituting lectio divina and deep listening as a new normal was essential having teachers replicate this process in their classrooms increased student engagement and by using poems by Langston Hughes and Rita Dove it created new opportunities to engage in discussions about racism and the possibilities for creating a greater sense of equity in the classroom. 


\section{Conclusion}

Over the pandemic, it was relationships between teachers and students which were critically important. Technology was the conduit for creative connection. The separate entities of SEL and technology must now be integrated (Bonanni et al., 2006). Our pandemic pivot examples bring technology together in concert with SEL in response to the needs of our urban school partners. Responding to the critical needs of students and teachers, our pandemic pivots represent a means to begin answering the call for holistically integrating SEL and technology and then maintaining those integrations post pandemic.

Our integrated TPP stands alongside this conceptual integration of SEL and technology. Almeida and Hall (2021b), write about this same school-university partnership and characterize it as "bidirectional" and symbiotic in the best ways. They add that "Together, the university and the school collaboratively design and embed elements that positively impact teacher retention and effectiveness" (p. 172). In responding to our partners call for connecting SEL and technology in different pedagogical ways, we demonstrate our progress and visualize next steps. Evaluation of the impact of these pandemic pivots and the implementation of this new normal is underway. Initial qualitative interviews with ICs have determined they have reviewed the performance of the in-service candidates in the TPP and they found that our candidates had a significant impact on their classroom students when compared to ways their peers integrated technology and SEL. Also, the TEAC was also surveyed in the summer of 2021 examining the impact of the pivot. Responses from the TEAC indicated overwhelming support for the new normal and where possible, looking for opportunities to expand. Quantitatively, in-service teachers in the TPP have asked their students to rate their effectiveness multiple times over the semesters of fall 2020 and spring 2021. Using repeated ANOVA analysis, we seek to understand the impact of these practices as perceived by students in urban classrooms. Data and study results continue to be measured and readied for publication in 2022.

What is not completely clear is whether this pandemic has just shown a light on the persistent inequities or provided unique opportunities to address the root causes of these persistent equities. What is true is that our work with partner schools continues. We strive for innovation in our approaches to teacher education and understand how important it is to deepen our relationships with the schools and our university. We seek dynamic ways to serve as an anchor for our teachers and the larger community. The true pandemic pivot was the centralization of our urban partners' voices and the new normal is an improved TPP meeting the dynamic needs of those partners.

(C) The Author 2022. Published by the Coalition of Urban and Metropolitan Universities. www.cumuonline.org 


\section{References}

Almeida, T., \& Hall, M. P. (2021a). An Untapped Resource: Embedded School Personnel in University-Based Teacher Preparation Programs as a High-Impact Practice. In B. Zugelder (Ed.), Empowering Formal and Informal Leadership While Maintaining Teacher Identity (pp. 206-226). IGI Global. https://doi.org/10.4018/978-1-7998-6500$\underline{1 . \operatorname{ch} 008}$

Almeida, T. \& Hall, M. P. (2021b). Creating an even-playing field: One model for a sustainable and replicable school-university partnership. In Chandler, P. T. \& Barron, L. (Eds.), Rethinking School-University Partnerships: A New Way Forward. Information Age Publishers.

Ailwood, J., \& Lee, I. F. (2020). Care matters: Reimagining early childhood education and care in a time of global pandemic. Global Studies of Childhood. Vol. 10(4) 309-312.

Bifuh-Ambe, E., Hall, M.P., and Lopes-Mendes, T. (2020). Activating lectio divina as a transformative process for anti-racism engagement and empowerment. Learning \& Sharing Virtual Series. Coalition of Urban and Metropolitan Universities (CUMU), December 9, 2020. https://vimeo.com/489130772/6815b148a7

Bonanni, L., Vaucelle, C., Lieberman, J., \& Zuckerman, O. (2006, April). PlayPals: tangible interfaces for remote communication and play. In CHI'O6 extended abstracts on Human factors in computing systems (pp. 574-579).

Bonvin, G., \& Sanchez, E. (2017). Social engagement in a digital role-playing game dedicated to classroom management. In Lecture Notes in Computer Science (including subseries Lecture Notes in Artificial Intelligence and Lecture Notes in Bioinformatics) (Vol. 10653 LNCS, pp. 137-147). https://doi.org/10.1007/978-3-319-71940-5_13

Campbell, S., personal communication, October 25, 2020.

Clinton, C. (2019). The Role of Teacher Education Advisory Committees in Massachusetts Teacher Preparation. ProQuest Dissertations Publishing.

Dalton, J.E., Hall. M. P., \& Hoyser, C.E. (2019) (Eds.). The Whole Person: Embodying Teaching and Learning through Lectio and Visio Divina. Rowman \& Littlefield.

Dorman, E. H. (2019). Lectio divina as contemplative, anti-oppressive pedagogy in social justice education courses. In Dalton, J.E., Hall. M. P., \& Hoyser, C.E. (Eds), The Whole Person: Embodying Teaching and Learning through Lectio and Visio Divina. Rowman \& Littlefield.

Elias, M. J. (2018). Analysis of the alignment of Classcraft's SEL environment and CASEL SEL standards. Retrieved from www.secdlab.org 
Finning, C. personal communication, October 272020.

Goldhaber, D., \& Cowan, J. (2014). Excavating the teacher pipeline: Teacher preparation programs and teacher attrition. Journal of Teacher Education, 65(5), 449-462. https://doi.org/10.1177/0022487114542516

Jennings, P. A. (2015). Mindfulness for teachers: Simple skills for peace and productivity in the classroom (the Norton series on the social neuroscience of education). WW Norton \& Company.

Krieg, J. M., Theobald, R., \& Goldhaber, D. (2016). A Foot in the Door: Exploring the Role of Student Teaching Assignments in Teachers' Initial Job Placements. Educational Evaluation and Policy Analysis, 38(2), 364-388. https://doi.org/10.3102/0162373716630739

Lee, J. J. C. U., \& Hammer, J. C. U. (2011). Gamification in education: What, how, why bother? Academic Exchange Quarterly, 15(2).

Membrive, V., \& Armie, M. (2020). Beyond gamification: Classcraft as an engagement tool in the teaching of English as a second language. (pp. 73-76). https://doi.org/10.36315/2020end016

Rock, D. (2008). SCARF model: a brain-based model for collaborating with and influencing others. Neuroleadership Journal, 1-9.

Rock, D., \& Tang, Y. (2009). Neuroscience of engagement. NeuroLeadership Journal, 2, 15-22.

Rozhenko, O. D., Darzhaniya, A. D., Bondar, V. V., \& Mirzoian, M. V. (2021). Gamification of education as an addition to traditional educational technologies at the university. CEUR Workshop Proceedings, 2914, 457-464.

Tan, C. (2021). Mindful education for a post-pandemic world. In Mindful education (pp. 179197). Springer. 